\title{
Article
}

\section{Determination of the dynamic shearing force and bending moment of a tensioned single-walled carbon nanotube subjected to a uniformly distributed external pressure}

\author{
A. A. Yinusa ${ }^{1, *}$, M. G. Sobamowo ${ }^{1}$ and A. O. Adelaja ${ }^{1}$ \\ 1 Department of Mechanical Engineering, University of Lagos, Akoka, Lagos, Nigeria.; \\ mynotebook2010@yahoo.com(A.A.Y) mikegbeminiyi@gmail.com(M.G.S); aadelaja@unilag.edu.ng(O.A) \\ * Correspondence: mynotebook2010@yahoo.com
}

Received: 02 April 2019; Accepted: 10 September 2019; Published: 30 September 2019.

\begin{abstract}
The high strength-to-weight ratio and flexibility of single walled carbon nanotubes (SWCNT) make them of potential use in the control of nanoscale structures for thermal, electrical, structural and mechanical applications. This indicates that they will have a vital contribution to nanotechnology engineering. This paper presents an exact solution to the dynamic response of such CNTs considering the shear force and bending moment under uniformly distributed external pressure. The dynamic behaviour of the SWCNT is modeled by employing the theories of Euler-Bernoulli beam and thermal elasticity mechanics. The developed model that governs the physics of the behaviour of the SWCNT when excited by the aforementioned external agents is solved using Integral transforms. The results of the close form solution in this work were compared with results of past works and excellent agreements were achieved. Furthermore, the dynamic study revealed that a point of maximum shear force on the CNT produced the minimum bending moment at any mode and for any parameter value considered. It is envisaged that this work will enhance the application of SWCNT for structural, electrical and mechanical uses.
\end{abstract}

Keywords: Dynamic study, external uniform pressure, integral transform, single walled carbon nanotube.

\section{Introduction}

$\mathbf{S}$ ince the discovery of CNT by Iijima [1], a considerable number of studies on carbon nanotubes with multi branched arrangements have been analyzed [2-4]. Subsequently, it has been established that such structures have merits when applied to the functionability of transistors and diodes. As a result, logical investigations and analysis of carbon nanotube have been a subject of interest such as the vibrations of a micro-resonator that is excited by electrostatic and piezoelectric actuations. Studies have been performed on beams, CNT, nano-wires, nano-rods and nano-beam so as to specifically understand and achieve their area of best fit. In achieving this, the well known beam models were employed and dynamic ranges were obtained in the scope of the structures [5-8]. Different studies have been performed to determine the resonance point and to enhance the mechanical properties of single and multi-walled nanotubes, using initial stress and compressive axial loading, to study the non-coaxial resonance [9-14]. Elishakoff et al., [15] performed an investigation on the fundamental natural frequencies of double-walled carbon nanotubes and ascertained some stability criteria using some controlling parameters. Mass detection with nonlinear nano-mechanical resonator as well as dynamic range of nanotube- and nanowire-based electromechanical systems have also been studied [16,17].

In order to be able to predict the dynamic behaviour of embedded carbon nanotubes, Fu et al.[18] and $\mathrm{Xu}$ et al. [19] performed some analysis of nonlinear vibration for embedded carbon nanotubes. In application to electrical and electronics, the static and dynamic analysis of carbon nanotube-based switches, micro-resonator under piezoelectric and electrostatic actuations have been presented [20-22]. As a continuation of the tremendous work, Abdel-Rahman and Nayfeh [23], Hawwa and Al-Qahatani [24] and, Hajnayeb and Khadem [25] performed vibration and instability studies of double wall carbon natotube (DWCNT) using a nonlinear model and considering electrostatic actuation as external excitation agent. In their investigation, DWCNT 
was situated and conditioned to direct and alternating voltages and different behaviors of the nanotubes were recorded as the exciting agent was varied. They went further to determine the bifurcation point of the DWCNT and concluded that both walls had the same frequency of vibration under the two resonant conditions considered. Belhadj et al. [26] presented their work on a pinned-pinned supported SWCNT employing nonlocal theory of elasticity and obtained natural frequency up to the third mode. They also explained how the high frequency obtained in their work might be harnessed to be of high merit in optical applications. Lei et al. [27] studied the dynamic behaviour of DWCNT by employing the well-known Timoshenko beam theory. The nonlinear governing equations obtained by Sharabiani and Yazdi [28] were applied to nanobeams that were graded and had surface roughness. Wang [29] obtained a closed form model for the aforementioned surface roughness effect for an unforced fluid conveying nanotube and beams based on nonlocal theory of elasticity and ascertained the significance of stability analysis for reasonably small thickness of the tube considered.

Interesting foundation studies have been considered after modelling of CNTs as structures resting on or embedded in elastic foundations such as Winkler, Pasternak and Visco-Pasternak media [30-35]. Other interesting works through modelling and experiment have also been presented to justify the widespread application of SWCNTs [36-41]. To the best of the authors' knowledge, no work has been done to dynamically determine the shearing force and bending moment diagram of a tensioned and pressurized SWCNT. Motivated by these considerations, this work aims to dynamically determine the response, shearing force and bending moment of a tensioned SWCNT under an external uniform pressure by employing Integral transform techniques.

\section{Problem description and governing equation}

Consider a SWCNT with a uniformly distributed surface pressure as shown in Figure 1. The assumptions used for the analysis include:

1. the SWCNT is modeled according to Euler-Bernoulli beam

2. the exciting agent is a uniform pressure distribution at the SWCNT surface governed by:

$$
P(x)=\mu A^{C N T} \frac{d}{d x}\left(P_{0}\left(1+\frac{\delta}{L^{C N T}} x\right)\right)
$$

3. the SWCNT is simply supported at both ends.

$$
\begin{array}{r}
\chi(0, t)=\chi^{\prime \prime}(0, t)=0 \\
\chi\left(L^{C N T}, t\right)=\chi^{\prime \prime}\left(L^{C N T}, t\right)=0
\end{array}
$$

4. CNT is homogenous and have constant properties.

5. CNT have constant cross-section, hence constant moment of Inertia.

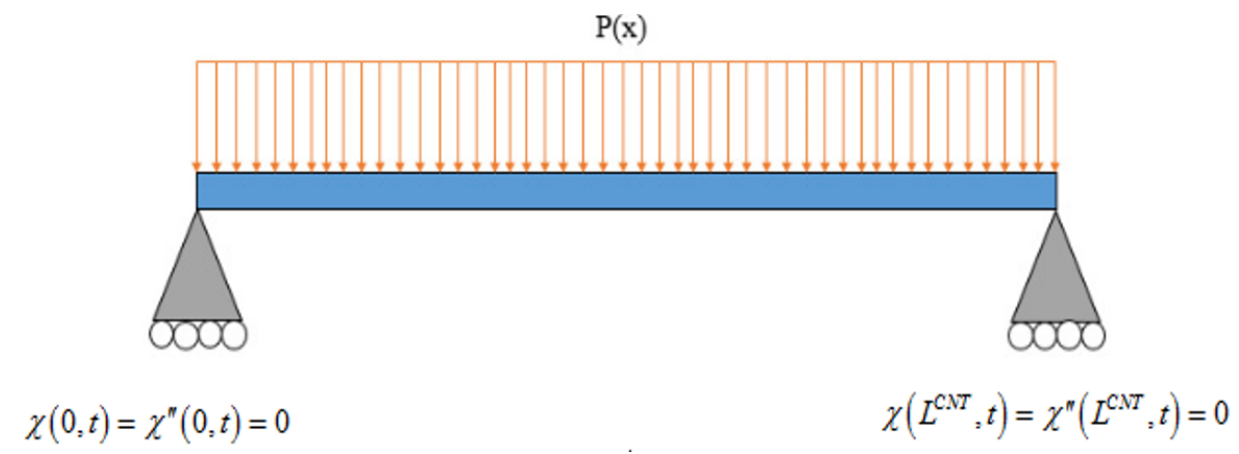

Figure 1. Schematic of the SWCNT with Static and Moving uniformly distributed pressure at the surfaces

By incorporating the above assumptions into the classic Euler-Bernoulli beam model, the vibration of Figure 1 can be described by the model:

$$
E I^{C N T} \frac{\partial^{4} \chi}{\partial x^{4}}+\left(\frac{E A}{1-2 v *} \alpha^{*} \theta-T\right) \frac{\partial^{2} \chi}{\partial x^{2}}+M \frac{\partial^{2} \chi}{\partial t^{2}}+K \chi=P(x)
$$


Substituting Equation (1) into Equation (3) and simplifying, the model becomes:

$$
E I^{C N T} \frac{\partial^{4} \chi}{\partial x^{4}}+\left(\frac{E A}{1-2 v *} \alpha^{*} \theta-T\right) \frac{\partial^{2} \chi}{\partial x^{2}}+M \frac{\partial^{2} \chi}{\partial t^{2}}+K \chi=\mu A^{C N T} \frac{d}{d x}\left(P_{0}\left(1+\frac{\delta}{L^{C N T}} x\right)\right)
$$

\section{Methods of solution using integral transforms}

Since the resulting model in Equation (4) contains both spatial and temporal part, a superb approach of applying Laplace transform to the temporal and Fourier transform to the spatial terms is adopted. In this present study, the linear transient model in Equation (3) will be solved exactly using Integral transform.

\subsection{Basic principle of the integral transforms}

Fourier transforms can be used to transform second and higher-order spatial derivatives. Although, the inversion of Fourier transform is usually easier than the inversion of Laplace transform, there are some functions which do not have Fourier transform as they have in Laplace transform. Fourier transform could be used to solve problems in.

1. Finite domains $(0 \leq x \leq L)$

2. Semi-infinite domain, and $(0 \leq x \leq \infty,-\infty \leq x \leq 0)$

3. Infinite domains unlike Laplace transform $(-\infty \leq x \leq \infty)$

The transform of a finite fourier cosine function is:

$$
F_{c}=\bar{f}(n)=\int_{0}^{l} \cos \left(\frac{n \pi x}{L}\right) f(x) d x \quad 0 \leq x \leq L, \text { where } n=\text { index counter } 1,2,3, \ldots
$$

The operational properties of the finite fourier cosine function are:

$$
\begin{array}{r}
F_{\mathcal{C}}\left[\frac{d f}{d x}\right]=-(1)^{n} f(l)-f(0)+\frac{n \pi}{L} \bar{f}_{s}(n) \\
F_{C}\left[\frac{d^{2} f}{d x^{2}}\right]=-(1)^{n} \frac{d f}{d x_{x=L}}-\frac{d f}{d x_{x=0}}-\frac{n^{2} \pi^{2}}{L^{2}} \bar{f}_{s}(n)
\end{array}
$$

Generally,

$$
\begin{gathered}
F_{c}\left[\frac{d^{2 N} f}{d x^{2 N}}\right]=(-1)^{n} \beta^{2 n} \bar{f}_{c}-\sum_{m=0}^{n-1}(-1)^{m} \beta^{2 m} \frac{\partial^{2 n-2 m-1} f\left(0^{+}\right)}{\partial x^{2 n-2 m-1}} \text { for } n \geq 1 \text { provided that }\left|\frac{d^{m} f}{d x^{m}}\right| \rightarrow 0 \text { as } x \rightarrow \infty \\
{\left[\frac{d^{2 n+1} f(x)}{d x^{2 n+1}}\right]=(-1)^{n} \beta^{2 n+1} \bar{f}_{s}-\sum_{m=1}^{n}(-1)^{m} \beta^{2 m} \frac{\partial^{2 n-2 m} f\left(0^{+}\right)}{\partial x^{2 n-2 m}} n \geq 1 .}
\end{gathered}
$$

The finite Fourier cosine inverse transform is:

$$
F_{c}^{-1}[f \bar{f}(n)]=f(x)=\frac{\bar{f}(n=0)}{L}+\frac{2}{L} \sum_{n=1}^{\infty} \bar{f}(n) \cos \left(\frac{n \pi x}{L}\right) .
$$

The finite Fourier sine transform of a function is defined as:

$$
\left.F_{s}[f(n)]=f \bar{x}\right)=\int_{0}^{l} f(x) \sin \left(\frac{n \pi x}{L}\right) \quad 0 \leq x \leq L . n=1,2,3, \ldots
$$

The operational properties of the sine transform of a function are:

$$
F_{S}\left[\frac{d f}{d x}\right]=-\frac{n \pi}{L} \bar{f}_{c}(n)
$$




$$
\begin{gathered}
F_{S}\left[\frac{d^{2} f}{d x^{2}}\right]=-\frac{n \pi}{L}\left[f(0)-(-1)^{n} f(L)\right]-\frac{n^{2} \pi^{2}}{L^{2}} \bar{f}_{c}(n) \\
F_{S}\left[\frac{d^{3} f}{d x^{3}}\right]=-\left[\frac{n \pi}{L}\right]^{3} \bar{f} .
\end{gathered}
$$

Generally, we have

$$
F_{S}\left[\frac{d^{2 N} f}{d x^{2 N}}\right]=(-1)^{n} \beta^{2 n} f_{s}+\sum_{m=1}^{n}(-1)^{m+1} \beta^{2 m-1} \frac{\partial^{2 n-2 m} f\left(0^{+}\right)}{\partial x^{2 n-2 m}}
$$

Thus, if the boundary value problem of a 2nd-order derivative extends over a finite domain and has 1st-type boundary condition at both ends $\left[f(0)=f_{0}\right.$ at $x=0, f(L)=f_{L}$ at $\left.x=L\right]$, the finite Fourier sine transform can be used to transform the 2 nd- order derivatives.

The finite Fourier sine inverse transform is:

$$
F_{c}^{-1}[\bar{f}(n)]=f(x)=\frac{2}{L} \sum_{n=1}^{\infty} \bar{f}(n) \sin \left[\frac{n \pi x}{L}\right] \quad 0 \leq x \leq L
$$

\subsection{Method of solution: Laplace and Fourier transform}

Recall that the linear transient governing equation as shown in Equation (4) may be expressed as

$$
M \frac{\partial^{2} \chi}{\partial t^{2}}+E I^{C N T} \frac{\partial^{4} \chi}{\partial x^{4}}+\left[\frac{E A}{1-2 v^{*}} \alpha^{*} \theta-T\right] \frac{\partial^{2} \chi}{\partial x^{2}}+K \chi=\mu A^{C N T} P_{0} \frac{\delta}{L^{C N T}}
$$

subject to the initial and pinned-pinned conditions:

$$
\begin{array}{r}
\chi(x, 0)=\dot{\chi}(x, 0)=0, \\
\chi(0, t)=\chi^{\prime \prime}(0, t)=0, \\
\chi\left(L^{C N T}, t\right)=\chi^{\prime \prime}\left(L^{C N T}, t\right)=0 .
\end{array}
$$

Applying Laplace transform on the temporal term gives

$$
M\left[s^{2} \bar{\chi}(x, s)-s \chi(x, 0)-\dot{\chi}(x, 0)\right]+E I^{C N T} \frac{d^{4} \bar{\chi}}{d x^{4}}\left[\frac{E A}{\left(1-2 V^{*}\right)} \alpha^{*} \theta-T\right] \frac{d^{2} \bar{\chi}}{d x^{2}}+K \chi=\mu A^{C N T} P_{0} \frac{\delta}{s L^{C N T}} .
$$

Using the initial condition and applying Fourier transform to the spatial terms, we have

$$
\begin{aligned}
& \mu A^{C N T} P_{0} \frac{\delta}{s L^{C N T}}\left(\frac{L^{C N T}}{i \pi}\right)\left[1-(-1)^{i}\right]=M s^{2} \widetilde{\bar{\chi}}+E I^{C N T}\left(\left(\frac{i \pi}{L^{C N T}}\right)^{4} \widetilde{\bar{\chi}}-\frac{i \pi}{L^{C N T}}\right)^{3}[\chi(0) \\
& \left.-(-1)^{i} \chi\left(L^{C N T}\right)\right]+\frac{i \pi}{L^{C N T}}\left[\chi^{\prime \prime}(0)-(-1)^{i} \chi^{\prime \prime}\left(L^{C N T}\right)\right]+\left[\frac{E A}{\left(1-2 V^{*}\right)} \alpha^{*} \theta-T\right]\left[-\left(\frac{i \pi}{L^{C N T}}\right)^{2} \widetilde{\bar{\chi}}\right. \\
& \left.+\frac{i \pi}{L^{C N T}}\right]\left[\chi(0)-(-1)^{i} \chi\left(L^{C N T}\right)\right]+K \widetilde{\bar{\chi}}
\end{aligned}
$$

Applying the boundary conditions and grouping like terms

$$
\tilde{\bar{\chi}}=\frac{\mu A^{C N T} P_{0} \delta\left(\frac{1}{i \pi}\right)\left[1-(-1)^{i}\right]}{s\left[M s^{2}+E I^{C N T}\left(\frac{i \pi}{L^{C N T}}\right)^{4}-\left[\frac{E A}{\left(1-2 V^{*}\right)} \alpha^{*} \theta-T\right]\left(\frac{i \pi}{L^{C N T}}\right)^{2}+K\right]} .
$$

\subsection{Determination of the natural frequency}

From Equation (12), the denominator may be arranged to take the form: 


$$
\begin{aligned}
\widetilde{\chi} & =\frac{\mu A^{C N T} P_{0} \delta\left(\frac{1}{i \pi}\right)\left[1-(-1)^{i}\right]}{s\left[s-j\left[\frac{I}{M}\left[E I^{C N T} \frac{i \pi}{L^{C N T}}\right]^{4}-\left[\frac{E A}{\left(1-2 V^{*}\right)} \alpha^{*} \theta-T\right]\right]\left(\frac{i \pi}{L^{C N T}}\right)^{2}+K\right]} \\
& \frac{\mu A^{C N T} P_{0} \delta\left(\frac{1}{i \pi}\right)\left[1-(-1)^{i}\right]}{\left[s+j\left[\frac{I}{M}\left[E I^{C N T} \frac{i \pi}{L^{C N T}}\right]^{4}-\left[\frac{E A}{\left(1-2 V^{*}\right)} \alpha^{*} \theta-T\right]\right]\left(\frac{i \pi}{L^{C N T}}\right)^{2}+K\right]} .
\end{aligned}
$$

The natural frequencies can be determined from the poles of Equation (12) as:

$$
\omega_{i}=\sqrt{\left\{\frac{1}{M}\left[E I^{C N T}\left(\frac{i \pi}{L^{C N T}}\right)^{4}-\left[\frac{E A}{\left(1-2 V^{*}\right)} \alpha^{*} \theta-T\right]\left(\frac{i \pi}{L^{C N T}}\right)^{2}+K\right]\right\}}
$$

The displacement of the nanotube will be realized by finding inverse Laplace transform of Equation (12). That is

$$
\begin{aligned}
& \tilde{\chi}=\frac{\mu A^{C N T} P_{0} \delta\left(L^{C N T}\right)^{4}}{i \pi\left(E I^{C N T}(i \pi)^{4}-\left(\frac{E A}{1-2 v^{*}} \alpha^{*} \theta-T\right)\left(i \pi L^{C N T}\right)^{2}+K\left(L^{C N T}\right)^{4}\right)} \\
& \times \frac{\left[1+(-1)^{i+1}+\cosh \left\{\frac{\sqrt{\left(L^{C N T}\right)^{4} M\left(-E I^{C N T}(i \pi)^{4}+\left(\frac{E A}{1-2 v^{*}} *^{*} \theta-T\right)\left(i \pi L^{C N T}\right)^{2}-K\left(L^{C N T}\right)^{4}\right)} t}{\left(L^{C N T}\right)^{4} M}\right\} 1+(-1)^{i}\right]}{i \pi\left(E I^{C N T}(i \pi)^{4}-\left(\frac{E A}{1-2 v^{*}} \alpha^{*} \theta-T\right)\left(i \pi L^{C N T}\right)^{2}+K\left(L^{C N T}\right)^{4}\right)}
\end{aligned}
$$

and the Fourier inverse of Equation (15) becomes:

$$
\begin{aligned}
& \chi(x, t)=\frac{2}{L^{C N T}} \sum_{i=1}^{\infty} \frac{\mu A^{C N T} P_{0} \delta\left(L^{C N T}\right)^{4}}{i \pi\left(E I^{C N T}(i \pi)^{4}-\left(\frac{E A}{1-2 v^{*}} \alpha^{*} \theta-T\right)\left(i \pi L^{C N T}\right)^{2}+K\left(L^{C N T}\right)^{4}\right)} \\
& \times \frac{\left[1+(-1)^{i+1}+\cosh \left\{\frac{\sqrt{\left(L^{C N T}\right)^{4} M\left(-E I^{C N T}(i \pi)^{4}+\left(\frac{E A}{1-2 v^{*}} \alpha^{*} \theta-T\right)\left(i \pi L^{C N T}\right)^{2}-K\left(L^{C N T}\right)^{4}\right)} t}{\left(L^{C N T}\right)^{4} M}\right\} 1+(-1)^{i}\right]}{i \pi\left(E I^{C N T}(i \pi)^{4}-\left(\frac{E A}{1-2 v^{*}} \alpha^{*} \theta-T\right)\left(i \pi L^{C N T}\right)^{2}+K\left(L^{C N T}\right)^{4}\right)}
\end{aligned}
$$

Equation (16) is the desired exact solution that represents deflection of the SWCNT.

\subsection{Determination of the SWCNT Bending moment and Shear force}

The bending moment is related to the SWCNT deflection by [39]:

$$
B(x, t)=-E I^{C N T} \frac{\partial^{2} \chi}{\partial x^{2}}
$$

Similarly, the shear force is related to the SWCNT deflection by:

$$
S(x, t)=-E I^{C N T} \frac{\partial^{3} \chi}{\partial x^{3}}
$$


Substituting Equation (16) into Equations. $(17,18)$, we have

$$
\begin{aligned}
& B(x, t)=-E I^{C N T} \frac{\partial^{2}}{\partial x^{2}}\left[\frac{2}{L^{C N T}} \sum_{i=1}^{\infty} \frac{\mu A^{C N T} P_{0} \delta\left(L^{C N T}\right)^{4}}{i \pi\left(E I^{C N T}(i \pi)^{4}-\left(\frac{E A}{1-2 v^{*}} \alpha^{*} \theta-T\right)\left(i \pi L^{C N T}\right)^{2}+K\left(L^{C N T}\right)^{4}\right)} \sin \frac{n \pi x}{L^{C N T}}\right] \\
& \times \frac{\left[1+(-1)^{i+1}+\cosh \left\{\frac{\sqrt{\left(L^{C N T}\right)^{4} M\left(-E I^{C N T}(i \pi)^{4}+\left(\frac{E A}{1-2 v^{*}} \alpha^{*} \theta-T\right)\left(i \pi L^{C N T}\right)^{2}-K\left(L^{C N T}\right)^{4}\right)} t}{\left(L^{C N T}\right)^{4} M}\right\} 1+(-1)^{i}\right]}{i \pi\left(E I^{C N T}(i \pi)^{4}-\left(\frac{E A}{1-2 v^{*}} \alpha^{*} \theta-T\right)\left(i \pi L^{C N T}\right)^{2}+K\left(L^{C N T}\right)^{4}\right)} \\
& S(x, t)=-E I^{C N T} \frac{\partial^{3}}{\partial x^{3}}\left[\frac{2}{L^{C N T}} \sum_{i=1}^{\infty} \frac{\mu A^{C N T} P_{0} \delta\left(L^{C N T}\right)^{4}}{i \pi\left(E I^{C N T}(i \pi)^{4}-\left(\frac{E A}{1-2 v^{*}} \alpha^{*} \theta-T\right)\left(i \pi L^{C N T}\right)^{2}+K\left(L^{C N T}\right)^{4}\right)} \sin \frac{n \pi x}{L^{C N T}}\right] \\
& \times \frac{\left[1+(-1)^{i+1}+\cosh \left\{\frac{\sqrt{\left(L^{C N T}\right)^{4} M\left(-E I^{C N T}(i \pi)^{4}+\left(\frac{E A}{1-2 v^{*}} \alpha^{*} \theta-T\right)\left(i \pi L^{C N T}\right)^{2}-K\left(L^{C N T}\right)^{4}\right)} t}{\left(L^{C N T}\right)^{4} M}\right\} 1+(-1)^{i}\right]}{i \pi\left(E I^{C N T}(i \pi)^{4}-\left(\frac{E A}{1-2 v^{*}} \alpha^{*} \theta-T\right)\left(i \pi L^{C N T}\right)^{2}+K\left(L^{C N T}\right)^{4}\right)} .
\end{aligned}
$$

The bending moment using the series at $i=3$ which is the converged point becomes;

$$
\begin{aligned}
& B(x, t)=2 \frac{E I n^{2} \pi^{2}}{L^{3}}\left[\frac{\mu A P_{0} \varepsilon L^{3}}{\pi\left(E I \pi^{4}-\left(A P-N_{t}-T\right) L^{2} \pi+K L^{4}\right)}\right. \\
& \times\left[2-2 \cosh \left[\frac{\sqrt{\left(-E I \pi^{4}+\left(A P-N_{i}-T\right) L^{2} \pi^{2}-K L^{4}\right) L^{4} M}}{L^{4} M} t\right]\right] \\
& +\frac{1}{3} \frac{\mu A P_{0} \varepsilon L^{3}}{\pi\left(81 E I \pi^{4}-9\left(A P-N_{t}\right) L^{2} \pi^{2}+K L^{4}\right)}\left[2-2 \cosh \left[\frac{\sqrt{\left(-81 E I \pi^{4}+9\left(A P-N_{t}-T\right) L^{4} M\right)}}{L^{4} M} t\right]\right] \sin \frac{n \pi x}{L} .
\end{aligned}
$$

And the shear force becomes:

$$
\begin{aligned}
& S(x, t)=2 \frac{E I n^{3} \pi^{3}}{L^{4}}\left[\frac{\mu A P_{0} \varepsilon L^{3}}{\pi\left(E I \pi^{4}-\left(A P-N_{t}-T\right) L^{2} \pi+K L^{4}\right)}\right. \\
& \times\left[2-2 \cosh \left[\frac{\sqrt{\left(-E I \pi^{4}+\left(A P-N_{i}-T\right) L^{2} \pi^{2}-K L^{4}\right) L^{4} M}}{L^{4} M} t\right]\right] \\
& +\frac{1}{3} \frac{\mu A P_{0} \varepsilon L^{3}}{\pi\left(81 E I \pi^{4}-9\left(A P-N_{t}\right) L^{2} \pi^{2}+K L^{4}\right)}\left[2-2 \cosh \left[\frac{\sqrt{\left(-81 E I \pi^{4}+9\left(A P-N_{t}-T\right) L^{4} M\right)}}{L^{4} M} t\right]\right] \cos \frac{n \pi x}{L} .
\end{aligned}
$$

\section{Results and Discussion}

Figure 2 depicts the convergence criteria based on the number of iteration $(i)$ in the close form solution for the deflection of the simply supported SWCNT. The computational time associated with each iteration is shown in Table 1. It is clear that at $i=3$, the solution has already converged, hence, extending the iteration above three will only increase the computational time and cost with negligible effect on the improvement of the established solution.

\subsection{Effect of the modal number on the deflection of the SWCNT}

Figure 3 displays the simply supported SWCNT deflection along its length for the first five mode shapes. Critical visualization shows that as the modal number associated with the kernel that defines the boundary condition increases, the stability of the SWCNT under study decreases as a result of an increase in the cycles covered by the SWCNT for the same length. These occur because the kernel depends on the modal number. 


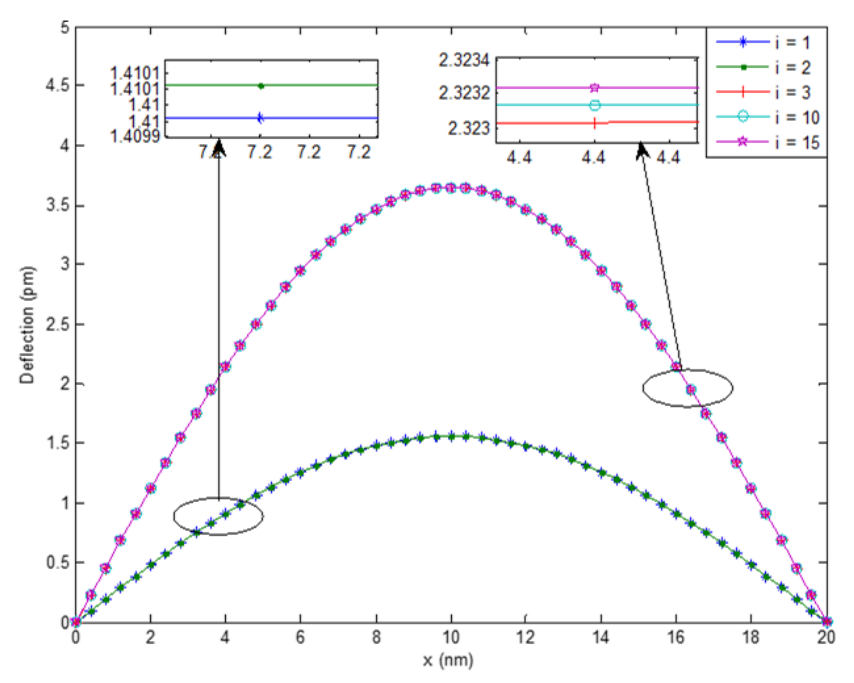

Figure 2. Convergence criteria

Table 1. Convergence criteria based on the number of iteration $(i)$ in the close form solution for the deflection of the SWCNT

\begin{tabular}{||cccccc||}
\hline $\begin{array}{c}\text { Iteration } \\
i\end{array}$ & chi $(\mathrm{pm})$ & $n=1$ & $\begin{array}{c}\text { Maximum deflection } \\
n=2\end{array}$ & $n=3$ & $\begin{array}{c}\text { Computational time (secs) } \\
n=4\end{array}$ \\
\hline \hline 1 & 1.600 & 0.256784 & 0.323885 & 0.435583 & 0.436961 \\
2 & 1.601 & 0.296788 & 0.323900 & 0.436781 & 0.446962 \\
3 & 3.700 & 0.456733 & 0.466385 & 0.468551 & 0.486001 \\
10 & 3.700 & 1.236734 & 1.523877 & 1.835101 & 1.999921 \\
15 & 3.700 & 3.456780 & 3.723335 & 3.935513 & 4.001610 \\
\hline
\end{tabular}

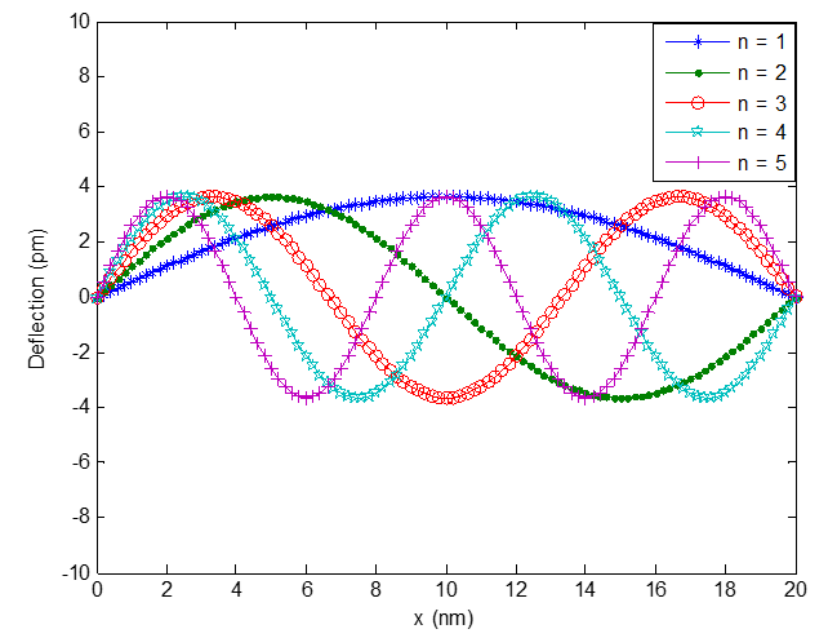

Figure 3. SWCNT modes with pinned-pinned condition

\subsection{Dynamic response of the SWCNT}

Figure 4, 5, 6, 7 depict the three dimensional dynamic response associated with the SWCNT for the first four modes. As the mode number increases, the number of cycles completed as shown by the dynamic behaviour of the system increases. A critical assessment shows that it is possible to track the behaviour of the CNT at any instance. The dynamic analysis is important as it helps in the quick monitory and adjustment of the CNT during application.

The model derived in the present study was reduced to the model of Coskun et al. [40] beam model and a very good agreement was obtained as shown in Table 2. 


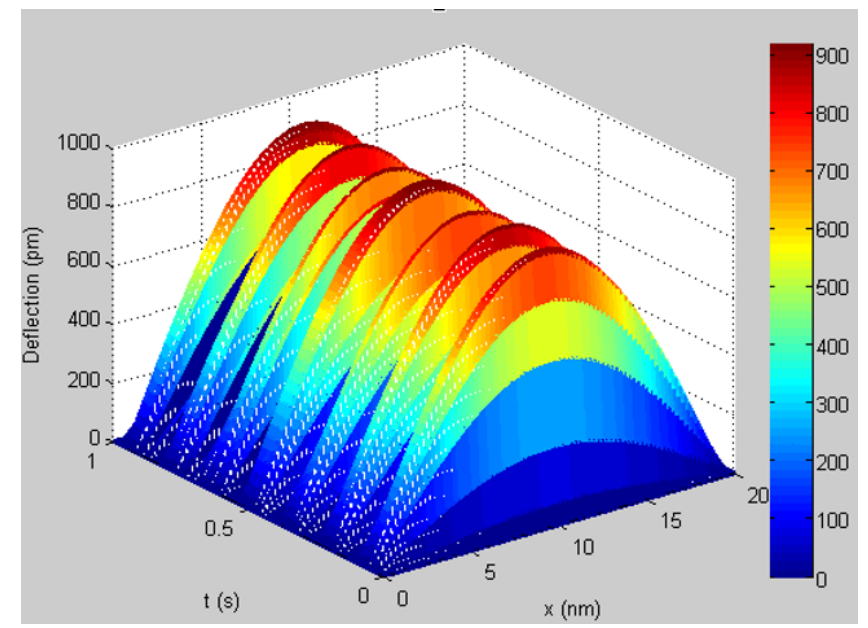

Figure 4. Dynamic response of the SWCNT for mode 1

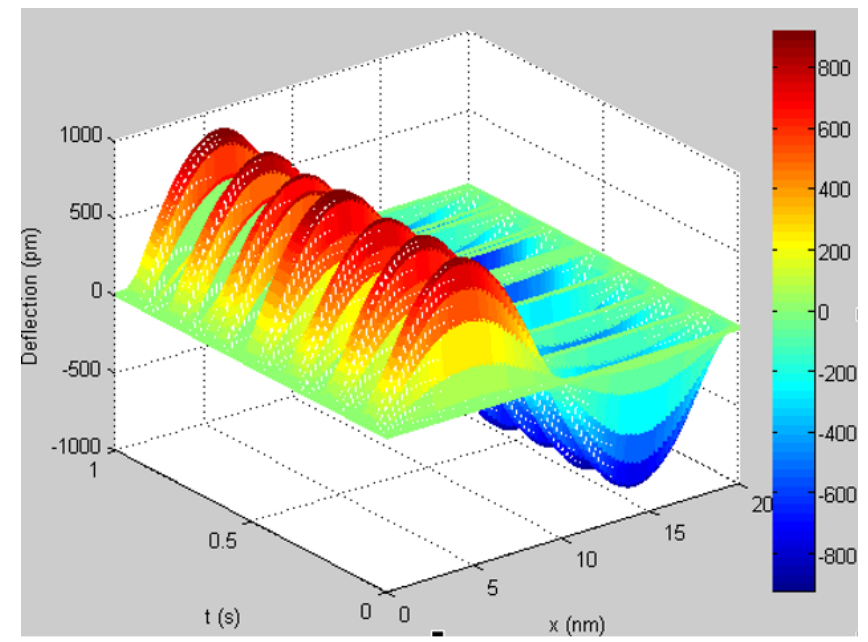

Figure 5. Dynamic response of the SWCNT for mode 2

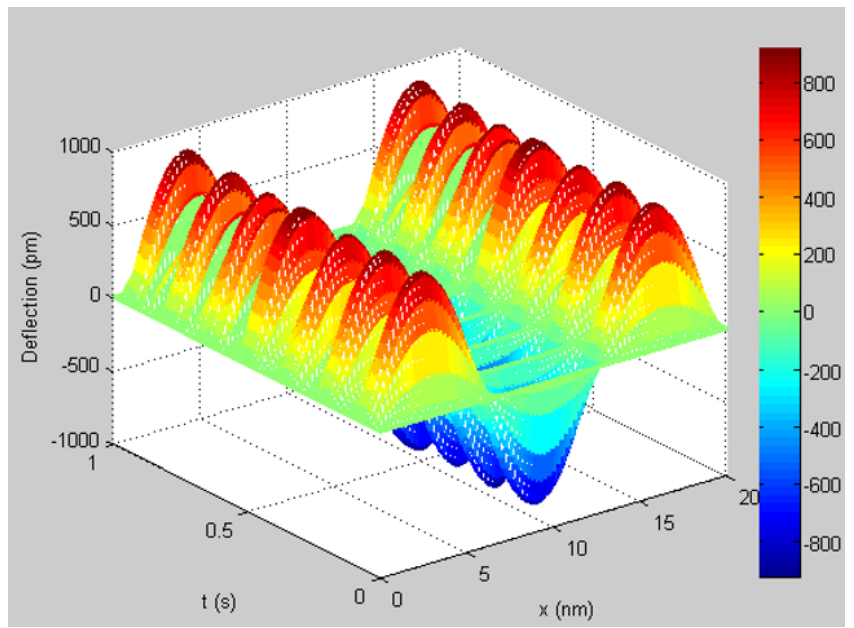

Figure 6. Dynamic response of the SWCNT for mode 3

\subsection{Effect of modal number and length on the frequency of the SWCNT}

Figure 8 depicts the influence of modal number and length on the frequency of the SWCNT. A careful study helps visualizes the effect of these two important parameters on stability of the SWCNT. The frequency of SWCNT which is a vital parameter in the study of the SWCNT stability reaches some THz and continues 


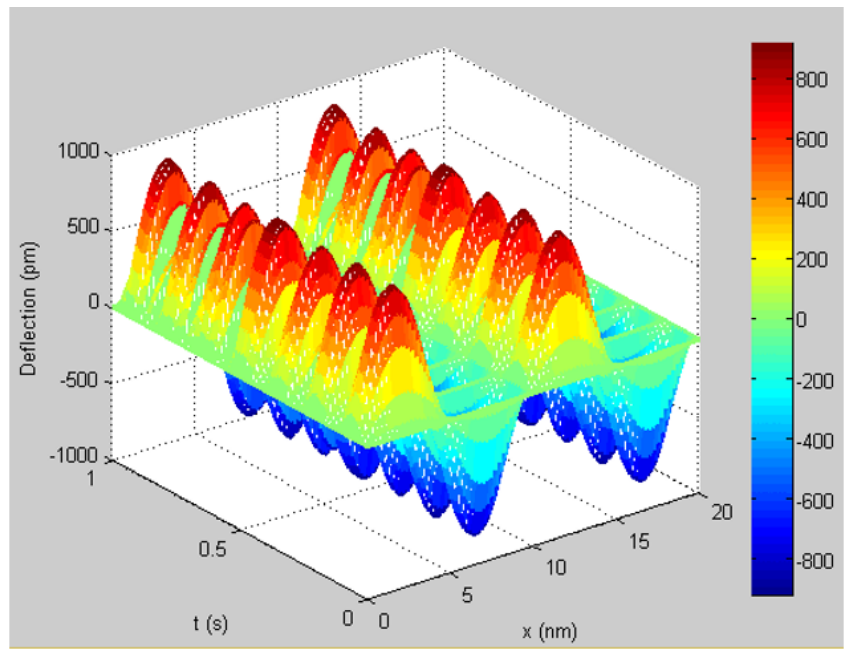

Figure 7. Dynamic response of the SWCNT for mode 4

Table 2. Comparison of present study with Coskun et al. [40] exact method for pinned - pinned condition

\begin{tabular}{|c|c|c|}
\hline Mode & $\begin{array}{c}\text { Mode shape } \\
\text { Coskun et al. [40] }\end{array}$ & Present study \\
\hline 1 & 3.14159265 & 3.14159265 \\
\hline 2 & 6.28318531 & 6.28318531 \\
\hline 3 & 9.42477796 & 9.42477796 \\
\hline 4 & - & 12.56637061 \\
\hline 5 & - & 15.70796327 \\
\hline
\end{tabular}

to increase as the modal number increases. This astonishing property enables SWCNT to offer exceptional optical and mechanical properties although there is always need to dampen the frequency to an application limit. These two parameters as a result of their tremendous effects on frequency may be used to annul the effect on each other when one of them is desired based on the requirement of the engineering design and applications. The dimensional frequency model obtained in the present study was also reduced to the level of

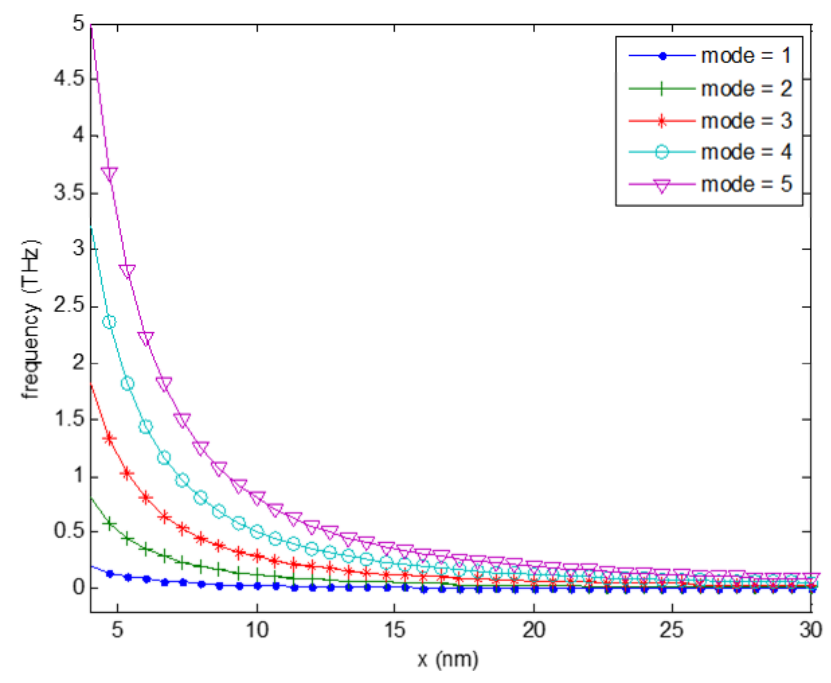

Figure 8. Effect of modal number on the frequency

Belhadj et al. [26] SWCNT model and a very good agreement was obtained for the first three modes as shown in Table 3. 
Table 3. Comparison of the present study with exact solution of Belhadj et al. [26] for pinned - pinned condition for the frequency of the SWCNT (THz)

\begin{tabular}{||ccccccc||}
\hline Length (nm) & Mode 1 & Mode 2 & $\begin{array}{c}\text { Belhadj et al. [26] } \\
\text { Mode 3 }\end{array}$ & Mode 1 & Mode 2 & $\begin{array}{c}\text { Present study } \\
\text { Mode 3 }\end{array}$ \\
\hline \hline 1 & 0.3 & 1.0 & 2.5 & 0.3 & 1.0 & 2.5 \\
2 & 0.2 & 0.6 & 1.6 & 0.2 & 0.6 & 1.6 \\
3 & 0.2 & 0.4 & 0.7 & 0.2 & 0.4 & 0.7 \\
4 & 0.2 & 0.3 & 0.5 & 0.2 & 0.3 & 0.5 \\
5 & 0.2 & 0.2 & 0.2 & 0.2 & 0.2 & 0.2 \\
\hline
\end{tabular}

\subsection{The Shear force and bending moment of the SWCNT}

Figure 9, 10, 11, 12 depict the three dimensional Shear force and bending moment diagram of the SWCNT for the first two modes. A critical assessment shows that it is possible to track the positions of maximum shear and maximum moment for proper design of the CNT device. The dynamic analysis is important as it helps in the quick monitory and adjustment of the CNT during application.

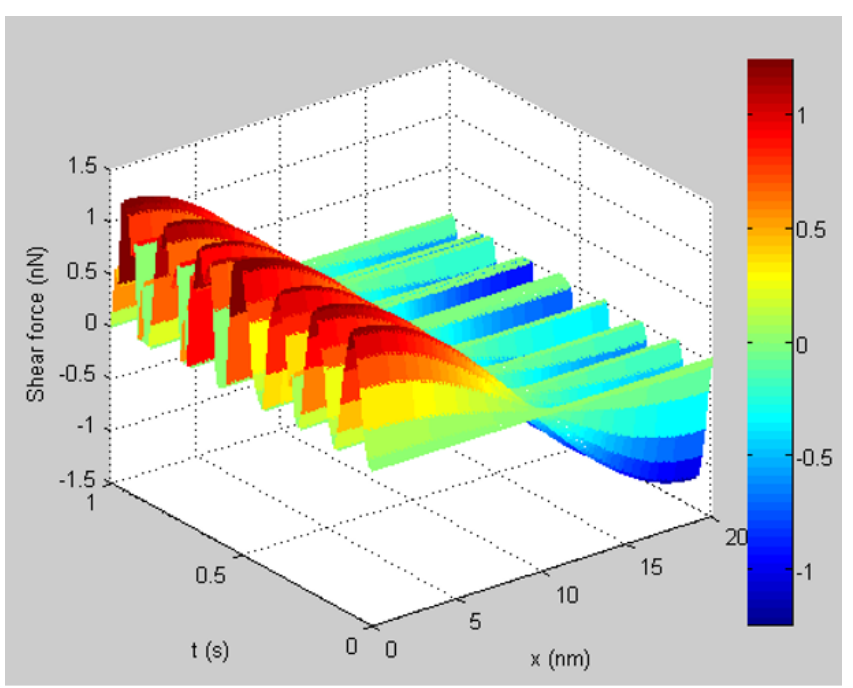

Figure 9. Shear force Diagram of the SWCNT for mode 1

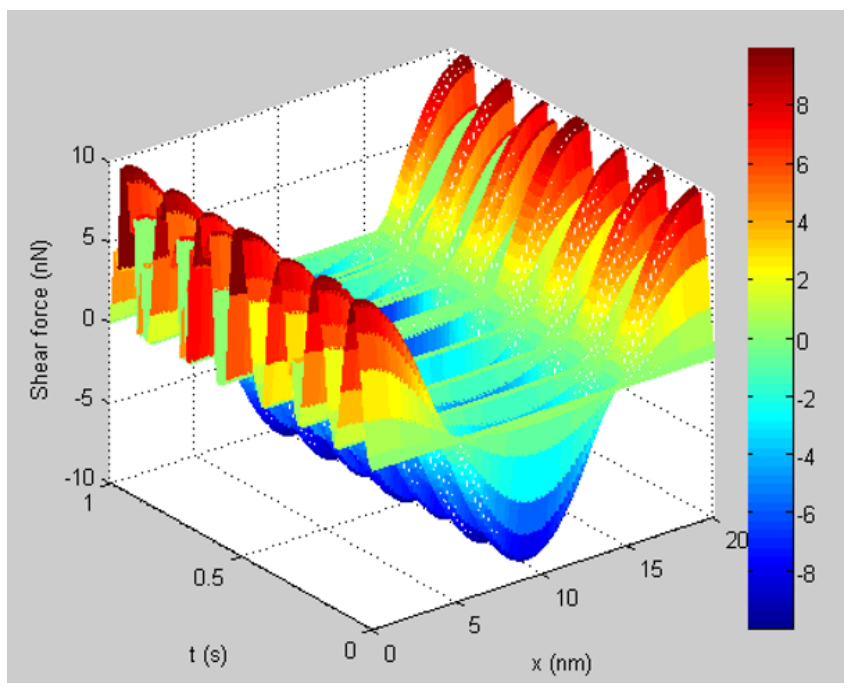

Figure 10. Shear force Diagram of the SWCN for mode 2 


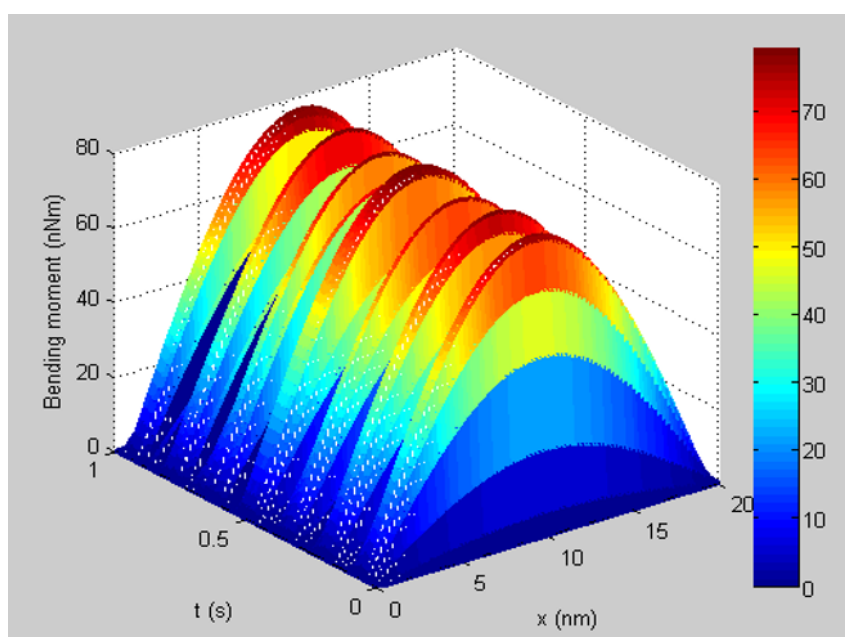

Figure 11. Bending moment Diagram of the SWCNT for mode 1

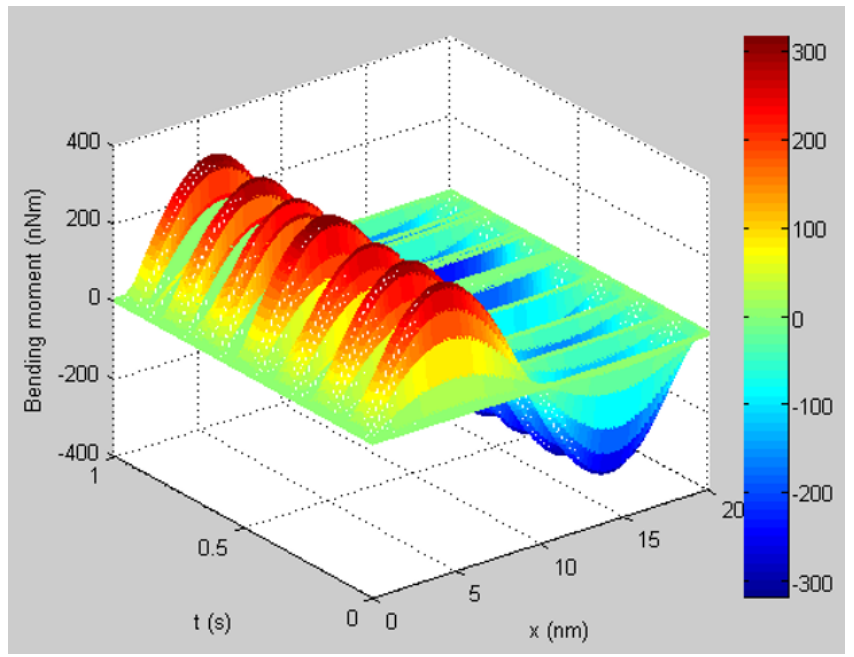

Figure 12. Bending moment Diagram of the SWCNT for mode 2

\section{Conclusion}

In this paper, analytical investigations of dynamic response of a SWCNT subjected to an external uniform pressure have been carried out using Integral transform. The exact solution as presented in the present study was reduced to the model of Coskun et al. [40] beam model and a very good agreement was obtained. The natural frequency as obtained in this study was also reduced to the model of Belhadj et al. [26] and an excellent agreement was reached. It was established that the Integral transform gave a good result and was efficient for the problem investigated. Also, some necessary parametric studies were performed to fully understand the dynamic behaviour of SWCNTs and to justify the widespread application of SWCNTs subjected to a uniformly distributed pressure acting externally. Furthermore, the dynamic study reveals that a point of maximum shear force on the CNT produces the minimum bending moment at any mode and for any parameter value considered. It is envisaged that this work will enhance the use of SWCNT under the influence of uniform surface pressure to structural, electrical and mechanical applications.

Author Contributions: All authors contributed equally to the writing of this paper. All authors read and approved the final manuscript.

Conflicts of Interest: "The authors declare no conflict of interest."

\section{References}

[1] Iijima, S. (1991). Helical microtubules of graphitic carbon. Nature, 354(6348), 56. 
[2] Terrones, M., Banhart, F., Grobert, N., Charlier, J. C., Terrones, H., \& Ajayan, P. M. (2002). Molecular junctions by joining single-walled carbon nanotubes. Physical review letters, 89(7), 075505.

[3] Nagy, P., Ehlich, R., Biro, L. P., \& Gyulai, J. (2000). Y-branching of single walled carbon nanotubes. Applied Physics A, 70(4), 481-483.

[4] Chernozatonskii, L. A. (1992). Carbon nanotube connectors and planar jungle gyms. Physics Letters A, 172(3), $173-176$.

[5] Liew, K. M., Wong, C. H., He, X. Q., Tan, M. J., \& Meguid, S. A. (2004). Nanomechanics of single and multiwalled carbon nanotubes. Physical review B, 69(11), 115429.

[6] Pantano, A., Boyce, M. C., \& Parks, D. M. (2004). Mechanics of axial compression of single and multi-wall carbon nanotubes. Journal of engineering materials and technology, 126(3), 279-284.

[7] Pantano, A., Parks, D. M., \& Boyce, M. C. (2004). Mechanics of deformation of single-and multi-wall carbon nanotubes. Journal of the Mechanics and Physics of Solids, 52(4), 789-821.

[8] Qian, D., Wagner, G. J., Liu, W. K., Yu, M. F., \& Ruoff, R. S. (2002). Mechanics of carbon nanotubes. Applied mechanics reviews, 55(6), 495-533.

[9] Salvetat, J. P., Bonard, J. M., Thomson, N. H., Kulik, A. J., Forro, L., Benoit, W., \& Zuppiroli, L. (1999). Mechanical properties of carbon nanotubes. Applied Physics A, 69(3), 255-260.

[10] Yinusa, A., \& Sobamowo, G. (2019). Analysis of Dynamic Behaviour of a Tensioned Carbon Nanotube in Thermal and Pressurized Environments. Karbala International Journal of Modern Science, 5(1), 2.

[11] Yoon, J., Ru, C. Q., \& Mioduchowski, A. (2002). Noncoaxial resonance of an isolated multiwall carbon nanotube. Physical Review B, 66(23), 233402.

[12] Wang, X., \& Cai, H. (2006). Effects of initial stress on non-coaxial resonance of multi-wall carbon nanotubes. Acta materialia, 54(8), 2067-2074.

[13] Wang, C. M., Tan, V. B. C., \& Zhang, Y. Y. (2006). Timoshenko beam model for vibration analysis of multi-walled carbon nanotubes. Journal of Sound and Vibration, 294(4-5), 1060-1072.

[14] Zhang, Y., Liu, G., \& Han, X. (2005). Transverse vibrations of double-walled carbon nanotubes under compressive axial load. Physics Letters A, 340(1-4), 258-266.

[15] Elishakoff, I., \& Pentaras, D. (2009). Fundamental natural frequencies of double-walled carbon nanotubes. Journal of Sound and Vibration, 322(4-5), 652-664.

[16] Buks, E., \& Yurke, B. (2006). Mass detection with a nonlinear nanomechanical resonator. Physical Review E, 74(4), 046619.

[17] Postma, H. C., Kozinsky, I., Husain, A., \& Roukes, M. L. (2005). Dynamic range of nanotube-and nanowire-based electromechanical systems. Applied Physics Letters, 86(22), 223105.

[18] Fu, Y. M., Hong, J. W., \& Wang, X. Q. (2006). Analysis of nonlinear vibration for embedded carbon nanotubes. Journal of Sound and Vibration, 296(4-5), 746-756.

[19] Xu, K. Y., Guo, X. N., \& Ru, C. Q. (2006). Vibration of a double-walled carbon nanotube aroused by nonlinear intertube van der Waals forces. Journal of Applied Physics, 99(6), 064303.

[20] Dequesnes, M., Tang, Z., \& Aluru, N. R. (2004). Static and dynamic analysis of carbon nanotube-based switches. Journal of engineering materials and technology, 126(3), 230-237.

[21] Ouakad, H. M., \& Younis, M. I. (2010). Nonlinear dynamics of electrically actuated carbon nanotube resonators. Journal of computational and nonlinear dynamics, 5(1), 011009.

[22] Zamanian, M., Khadem, S. E., \& Mahmoodi, S. N. (2009). Analysis of non-linear vibrations of a microresonator under piezoelectric and electrostatic actuations. Proceedings of the Institution of Mechanical Engineers, Part C: Journal of Mechanical Engineering Science, 223(2), 329-344.

[23] Abdel-Rahman, E. M., \& Nayfeh, A. H. (2003). Secondary resonances of electrically actuated resonant microsensors. Journal of Micromechanics and Microengineering, 13(3), 491.

[24] Hawwa, M. A., \& Al-Qahtani, H. M. (2010). Nonlinear oscillations of a double-walled carbon nanotube. Computational Materials Science, 48(1), 140-143.

[25] Hajnayeb, A., \& Khadem, S. E. (2012). Nonlinear vibration and stability analysis of a double-walled carbon nanotube under electrostatic actuation. Journal of Sound and Vibration, 331(10), 2443-2456.

[26] Belhadj, A., Boukhalfa, A., \& Belalia, S. A. (2017). Carbon Nanotube Structure Vibration Based on Non-local Elasticity. Journal of Modern Materials, 3(1), 9-13.

[27] Lei, X. W., Natsuki, T., Shi, J. X., \& Ni, Q. Q. (2012). Surface effects on the vibrational frequency of double-walled carbon nanotubes using the nonlocal Timoshenko beam model. Composites Part B: Engineering, 43(1), 64-69.

[28] Sharabiani, P. A., \& Yazdi, M. R. H. (2013). Nonlinear free vibrations of functionally graded nanobeams with surface effects. Composites Part B: Engineering, 45(1), 581-586. 
[29] Wang, L. (2010). Vibration analysis of fluid-conveying nanotubes with consideration of surface effects. Physica E: Low-dimensional Systems and Nanostructures, 43(1), 437-439.

[30] Biro, L. P., Horvath, Z. E., Mark, G. I., Osvath, Z., Koos, A. A., Benito, A. M., ... \& Lambin, P. (2004). Carbon nanotube Y junctions: growth and properties. Diamond and related materials, 13(2), 241-249.

[31] Lin, R. M. (2012). Nanoscale vibration characterization of multi-layered graphene sheets embedded in an elastic medium. Computational Materials Science, 53(1), 44-52.

[32] Pradhan, S. C., \& Phadikar, J. K. (2009). Small scale effect on vibration of embedded multilayered graphene sheets based on nonlocal continuum models. Physics letters A, 373(11), 1062-1069.

[33] Arani, A. G., Zarei, M. S., Amir, S., \& Maraghi, Z. K. (2013). Nonlinear nonlocal vibration of embedded DWCNT conveying fluid using shell model. Physica B: Condensed Matter, 410, 188-196.

[34] Arani, A. G., \& Amir, S. (2013). Electro-thermal vibration of visco-elastically coupled BNNT systems conveying fluid embedded on elastic foundation via strain gradient theory. Physica B: Condensed Matter, 419, 1-6.

[35] Eichler, A., Moser, J., Chaste, J., Zdrojek, M., Wilson-Rae, I., \& Bachtold, A. (2011). Nonlinear damping in mechanical resonators made from carbon nanotubes and graphene. Nature nanotechnology, 6(6), 339.

[36] Housner, G. W., Dodds, H. L., \& Runyan, H. (1965). Effect of High Velocity Fluid flow in the Bending Vibrations and static Divergence of Simply Supported Pipes. National Aeronautics and Space Administration Report NASA TN D-2870.

[37] Sobamowo, M. G. (2017). Nonlinear thermal and flow-induced vibration analysis of fluid-conveying carbon nanotube resting on Winkler and Pasternak foundations. Thermal Science and Engineering Progress, 4, 133-149.

[38] Liu, F., Wagterveld, R. M., Gebben, B., Otto, M. J., Biesheuvel, P. M., \& Hamelers, H. V. M. (2014). Carbon nanotube yarns as strong flexible conductive capacitive electrodes. Colloid and Interface Science Communications, 3, 9-12.

[39] Sobamowo, M. G., \& Yinusa, A. A. (2018). Thermo-fluidic parameters effects on nonlinear vibration of fluid-conveying nanotube resting on elastic foundations using homotopy perturbation method. J Therm Eng, 4(4), 2211-2233.

[40] Coskun, S. B., Atay, M. T., \& Ozturk, B. (2011). Transverse vibration analysis of Euler-Bernoulli beams using analytical approximate techniques. Advances in Vibration Analysis Research, 1-22.

[41] Sobamowo, M. G., \& Yinusa, A. A. (2017). Power Series-Aftertreatment Technique for Nonlinear Cubic Duffing and Double-Well Duffing Oscillators. Journal of Computational Applied Mechanics, 48(2), 297-306.

(c) 2019 by the authors; licensee PSRP, Lahore, Pakistan. This article is an open access article distributed under the terms and conditions of the Creative Commons Attribution (CC-BY) license (http://creativecommons.org/licenses/by/4.0/). 